\title{
The Difficult Neck in Facelifting
}

\author{
Fred G. Fedok, MD, FACS ${ }^{1,2}$ Irina Chaikhoutdinov, MD ${ }^{1}$ Frank Garritano, MD ${ }^{1}$
}

${ }^{1}$ Facial Plastic and Reconstructive Surgery, Division of Otolaryngology/ Head and Neck Surgery, Department of Surgery, Penn State Milton S. Hershey Medical Center, Hershey, Pennsylvania

2 The McCollough Plastic Surgery Clinic, Gulf Shores, Alabama

\begin{abstract}
Address for correspondence Fred G. Fedok, MD, FACS, Division of Otolaryngology/Head and Neck Surgery, Department of Surgery, Penn State Milton S. Hershey Medical Center, 500 University Drive, Hershey, PA 17033 (e-mail: ffedok@hmc.psu.edu).
\end{abstract}

Facial Plast Surg 2014;30:438-450.

\begin{abstract}
Keywords

- facelift

- rhytidectomy

- difficult neck

- liposuction

- SMAS

The management of the neck often presents the most challenging aspect of the facelift procedure. The aesthetic neck has a well-defined jaw line, a pleasing and adequate cervicomental angle, and visible definitions of the deeper lateral and midline structures, such as the sternocleidomastoid muscles and trachea. Several unfavorable anatomic characteristics will present that will compound the challenge. These characteristics are contrasted with ideal features and include the following: an excess of adipose tissue, an excess of either thin or thick inelastic skin, marked relaxation of the suspension structures of the neck with resultant platysma banding and jowling, and unfavorable skeletal features such as microgenia and hyoid malposition. These patients present for rhytidectomy with inadequate chin projection, an obtuse cervicomental angle, sagging skin, and a heavy neck. To maximize rhytidectomy results in these patients with difficult neck anatomy, special attention to the anatomy and application of recognized techniques in an individualized manner is recommended. This article reviews the issues encountered in the management of the difficult neck in facelifting, with special attention given to patients with a heavy neck.
\end{abstract}

The American Academy of Facial Plastic Surgery reports that the number of facelifts and blepharoplasty procedures continues to rise in the United States in its 2012 survey of members. ${ }^{1}$ While most of the increase in cosmetic procedures is attributed to nonsurgical methods, rhytidectomy continues to be among the most popular procedures. Approximately 46 rhytidectomy procedures on average were reported to have been performed by members in 2012 . The last several decades have seen the social acceptance of cosmetic and rejuvenative procedures growing, and a larger cross-section of the population undergoing surgery.

Not only those that are endowed with natural beauty and ideal facial characteristics and anatomy undergoing rhytidectomy, but a wide spectrum of patients with different anatomic attributes and ethnicities are also seeking surgery. Increased knowledge and technique development have identified the patients with favorable or ideal characteristics for undergoing rhytidectomy. ${ }^{2}$ Alternatively, patient characteristics that might be identified as less than ideal for rhytidectomy have also emerged. ${ }^{3}$ While these patients present a challenge for the surgeon in achieving a favorable result, they increasingly present for consultation and surgery. The purpose of this article is to investigate this concept of the difficult neck in rhytidectomy, to relate cues for identifying these patients, and to give recommendations for the management of these diverse patients' characteristics.

\section{Evolution of Rhytidectomy}

The earliest descriptions of rhytidectomy were based on elliptical skin excisions. Although these early aesthetic surgery techniques were frequently shrouded in secrecy, Julien Bourguet described his innovative approach to cervical rhytidectomy in 1919 in an address to the Academy of Medicine in Paris. He advocated for extensive undermining into the neck through incisions that extended behind the ear and into the hairline. He followed these contributions by describing the treatment of submental platysma banding by excision of offending platysma edges in $1936 .{ }^{4}$ Little additional advancement in neck-lifting surgery was described until 1974 when
Issue Theme Facets of Facelifting; Guest Editor, Daniel E. Rousso, MD, FACS
Copyright (C) 2014 by Thieme Medical Publishers, Inc., 333 Seventh Avenue, New York, NY 10001, USA. Tel: +1(212) 584-4662.
DOI http://dx.doi.org/ 10.1055/s-0034-1383554. ISSN 0736-6825. 
Skoog described a subplatysmal flap that elevated the platysma muscle of the neck and lower part of the face without detaching the skin. ${ }^{5}$ Mitz and Peyronie identified the superficial musculoaponeurotic system (SMAS) in 1976 as the anatomical basis behind Skoog's technique. ${ }^{6,7}$ The SMAS concept rapidly emerged as, and continues to be, central to facelifting technique.

During the same time period, adjunctive techniques to address the heavy neck and augment neck lifting were coming into vogue. In 1968, Millard et al described submental and submandibular lipectomy via short submental incisions to address the fatty neck in combination with face and neck lifting. ${ }^{8}$ Further advancement by Connell in 1968 described neck contouring using lipectomy combined with a muscle sling made from full-width platysma muscle flaps to provide deep support. ${ }^{9}$ Techniques to address the heavy neck expanded to include suction lipectomy of the neck, introduced by Courtiss in $1985 .{ }^{10}$ Management of the aging neck through the variable design of incisions, the application of open lipectomy, ultrasonic- and laser-assisted lipectomy, and suction-assisted lipectomy have also been investigated. ${ }^{11-15}$

Additional methods to improve the appearance of the platysma were reported. The corset platysmaplasty was described in $1990^{16}$ and joined a multitude of popular techniques including partial platysma muscle section, Zplasty techniques, and lateral or medial plication of the platysma. ${ }^{9,17-21}$ In 1997, Connell and Shamoun described the significance of digastric muscle contouring in neck lifting. ${ }^{22}$ In 2006, submandibular gland suspension to improve the appearance of the neck was described by Sullivan et al. ${ }^{23}$ This variety of methods for managing the anterior platysma bands, as well as the SMAS, have also been reported and are widely adopted. ${ }^{20,24-35}$ The deep plane technique has been advocated to address the heavy neck. ${ }^{36}$

As these techniques to address neck contouring and lifting evolved, several systems emerged for assessment and classification of neck appearance in the 1980s. Ellenbogen and Karlin described visual criteria for restoring the youthful neck, whereas the Dedo system graded suboptimal neck appearance based on presence of skin laxity, submental fat accumulation, platysma banding, retrognathia, and hyoid malposition. $^{37,38}$

The current literature on rhytidectomy is replete with techniques spanning the spectrum of traditional long-scar and short-scar surgeries. ${ }^{39-41}$ Among the recent innovations have been the development of several short-scar techniques.
A
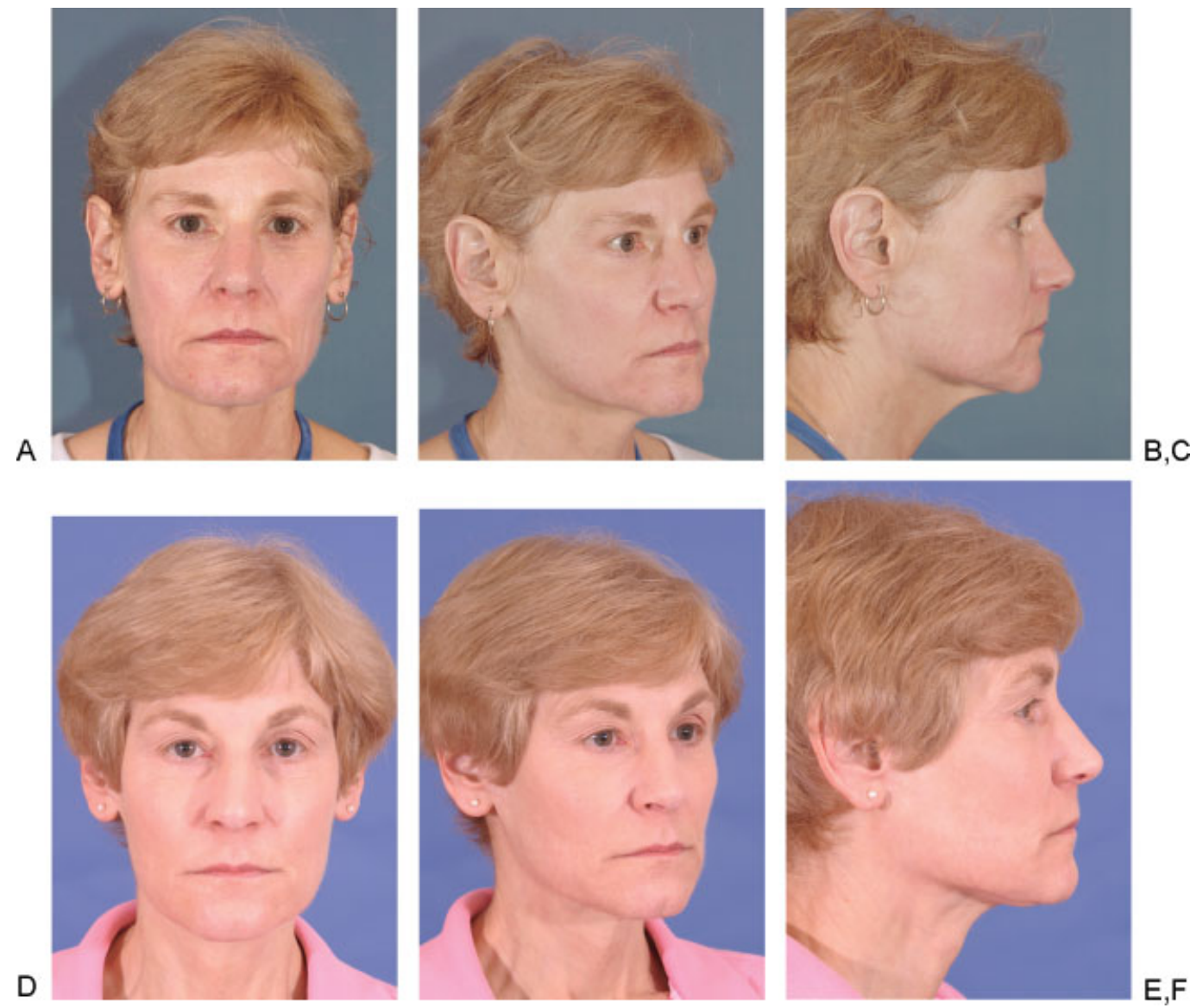

Fig. 1 (A-F) Clinical photographs of patient who exhibits anatomic characteristics favorable for short incision rhytidectomy, that is, favorable chin projection, favorable hyoid position, defined jaw line, limited skin excess, limited jowling, limited adipose; (A-C) preoperative photos; (D-F) postoperative photos. (G-I) Clinical photographs of more challenging patient who exhibits anatomic characteristics favorable for more traditional long incision rhytidectomy, that is, unfavorable chin projection, unfavorable hyoid position, less defined jaw line, moderate skin excess, significant jowling, significant adipose; (G-I) preoperative photos; (J-L) postoperative photos. 

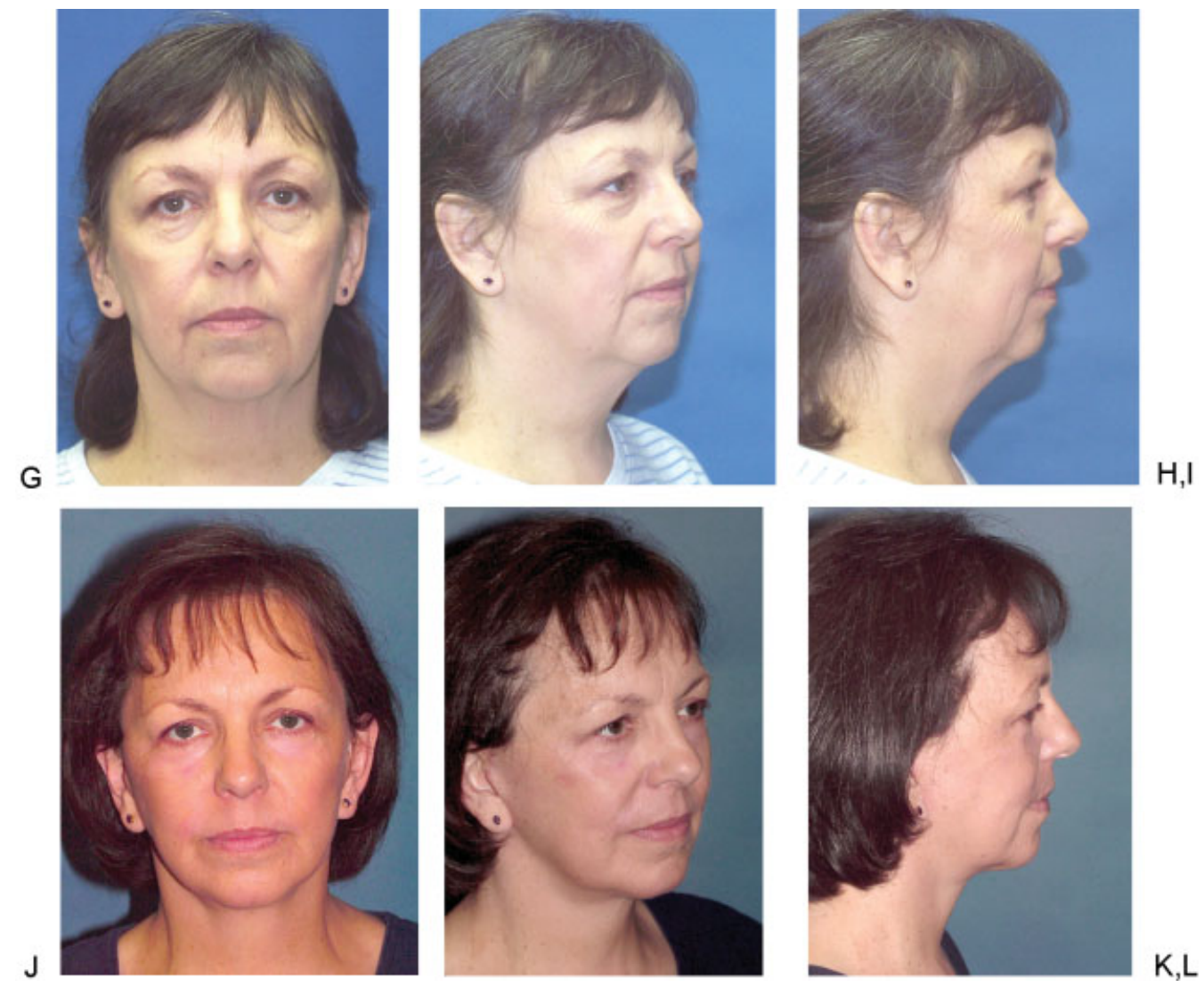

Fig. 1 (Continued)

These short scar techniques appear to have developed to accommodate patients' anatomic characteristics, their desire for less-invasive techniques, and economics. ${ }^{39,42,43}$ There is, at least, reasonable validity for the application of these techniques in properly selected patients. In general, the short-scar techniques appear to be best applied to those patients who require a lesser degree of skin management and have favorable skeletal features. ${ }^{41}$ In contrast, the patient with difficult neck anatomy is frequently best managed with a more traditional approach (-Fig. 1).

\section{Desirable Result in Facelifting}

The aesthetic neck has a well-defined jaw line, a pleasing and adequate cervicomental angle, and visible definitions of the deeper lateral and midline structures, such as the sternocleidomastoid muscles and trachea. ${ }^{44,45}$ There must be enough subcutaneous adipose to create smooth contours as one transitions from one structure to the other; there cannot be too little adipose that the neck looks skeletonized, nor excess that prevents the appreciation of deeper structures. There should be an absence of platysma bands, as well as skin laxity wrinkles and folds (-Fig. 2 ).

The achievement of a desirable surgical result is related to the patient's underlying attributes and the ability of the surgeon to correct or restore the patient's anatomy to that which imparts the characteristics of the ideal neck. Patients that bring the best attributes to the surgical table can gener- ally be expected to get the best results. The ideal patient has anatomic attributes that have many or all of the components of ideal esthetic neck anatomy. Those patients with more anatomic deficiencies are most likely to have more difficulty achieving an ideal result.

\section{Pertinent Anatomy of the Challenging Neck}

Excess adipose tissue in the neck leads to the appearance of the "heavy" neck and presents a challenge in neck-lifting surgery. Distribution of fat in the cervical area can be divided into three regions and can be either congenital or acquired. Adipose tissue can be diffusely distributed in the supraplatysmal layer throughout the cervical region. A submental fat collection between the anterior bellies of the digastric may be located subplatysmal and overlying the mylohyoid muscle. Lastly, adipose tissue may become displaced as result of the laxity of the platysma and attenuation of the mandibular ligament, creating a ptotic jowl with loss of definition of the inferior mandibular border. In addition to adipose tissue, prominent anterior border of the digastrics and ptotic submandibular glands may also detract from ideal cervical contours and contribute to the appearance of the heavy neck. Skin laxity with accumulation of subcutaneous fat can produce "turkey gobbler" deformity, making the neck appear heavy (-Fig. 3 ).

The two bellies of the platysma muscle, which lie deep in the subcutaneous tissue, originate from the fascia of the 
A

\section{B}

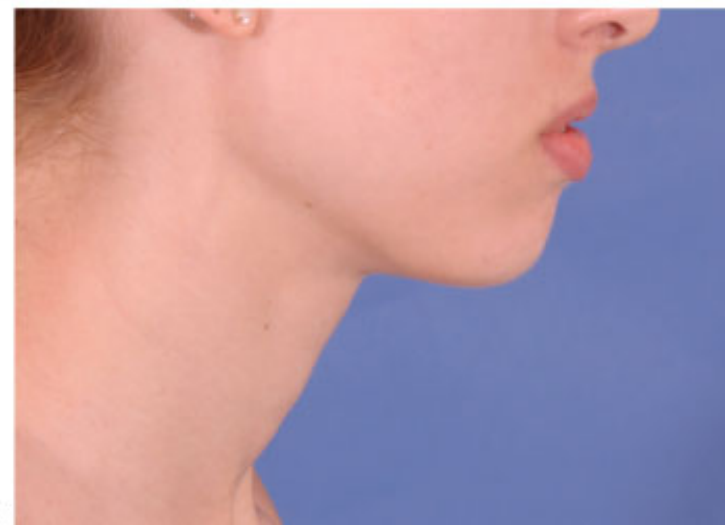

Fig. 2 (A, B) Clinical photograph of a patient with favorable neck anatomic features including: a well-defined jaw line, an adequate cervicomental angle, and visible definitions of the deeper lateral and midline structures.

pectoralis major and ascend vertically into the neck to insert at the inferior mandibular border. The platysma communicates with the SMAS of the face and is invested on both sides by the superficial cervical fascia. With aging, the platysma becomes atrophic and the platysmal sling is no longer able to support the underlying cervical contents, leading to submental soft tissue convexity. Loss of tone also results in platysma banding, well known to be associated with signs of aging.

The interdigitation pattern of the platysma contributes to the definition of the submental neck contour at the level of the hyoid and needs to be considered in surgical planning for patients seeking neck-lifting surgery. It is important to note that there are three anatomic variations pertaining to decussation of the platysma; in $10 \%$ of the population there is close approximation between the two bellies of the platysma with no decussation; in $75 \%$ of the population there is partial decussation in the midline; and in the remaining $15 \%$, total decussation from mandible to hyoid is present between the two bellies of the platysma. ${ }^{46}$ When the muscles decussate in the midline, an effective supportive sling exists in the submental area. When the decussation is absent, the free medial

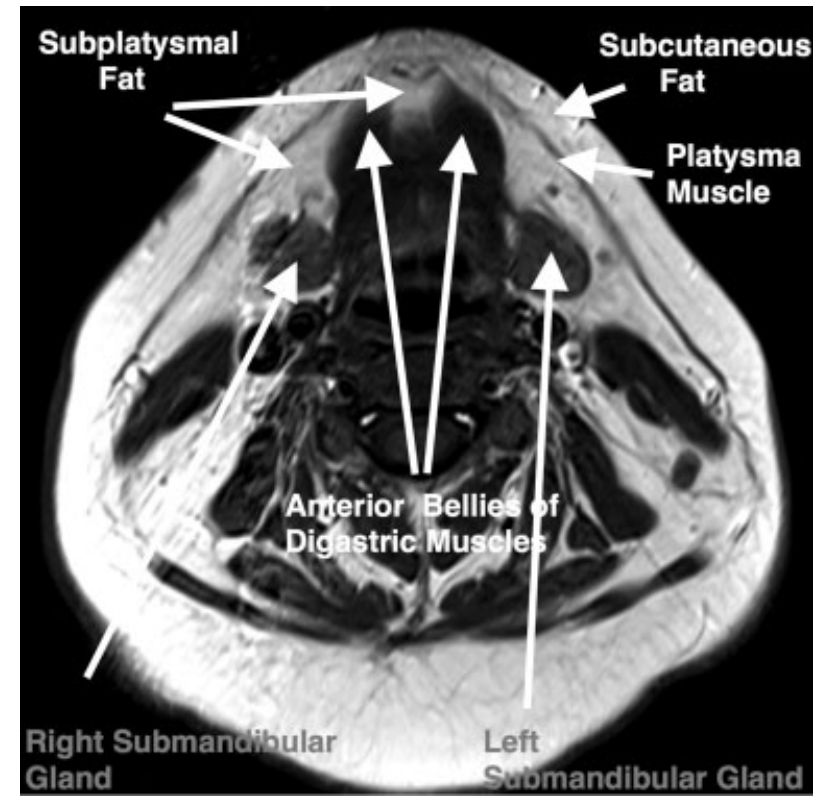

Fig. 3 Axial MRI image of patient's neck positioned below the mandibular body depicting subplatysmal and subcutaneous fat, submandibular glands, and anterior bellies of digastric muscles. (MRI image courtesy of Sangam Kanekar, MD, Department of Radiology, University Hospital, The Hershey Medical Center, The Pennsylvania State University.)

edges fall away from the submental area, and the patient is prone to form the anterior neck deformity known as "vertical bands." Addressing the platysma is key in obtaining a welldefined neck and jaw line.

The chin projection and hyoid position determine the cervicomental angle, which should ideally range from 90 to 105 degrees. The hyoid bone in the adult is ideally positioned at or above the level of the fourth cervical vertebra. Both anterior-posterior and superior-inferior positional variations of the hyoid are of analytical and prognostic value in cervical rejuvenation. A low and anteriorly positioned hyoid will produce an obtuse cervicomental angel by pulling the suprahyoid musculature in a more vertical course. Unfortunately, such anatomy will impose significant limitations on what can be achieved by typical cervicofacial rejuvenation methods.

Patients with weak or small chins can create a challenge in neck-lifting surgery. Underprojection of the chin, as in retrognathia with Angle's class II malocclusion, can contribute to a truncated jaw line. Microgenia, which describes an underprojected mentum independent of occlusal considerations, can similarly affect the jaw line. The ideal projection of the chin has been described by several different methods of assessment. In men, the pogonion is ideally tangent to a line drawn vertically from the lower vermilion border of the lip, and in women the pogonion ideally falls slightly posterior to this line. In a similar analysis described by Gonzalez-Ulloa, a vertical line perpendicular to the Frankfurt horizontal plane intersects the nasion, and the chin should be at or just posterior to this line. ${ }^{47}$ Although microgenia is most commonly congenital, mandibular hypoplasia secondary to 
absorption of alveolar bone with aging, results in formation of the "prejowl sulcus."

A high-positioned hyoid complex and well-defined facial bony characteristics comprise an anatomically ideal patient for neck lifting. In addition, good skin tone, lack of major platysma laxity or banding, and absent submental fat can contribute to optimal sculpting and definition of the jaw line. Unfortunately, certain anatomic variations can create challenges in cervical rejuvenation, making the ideal jaw line difficult to establish. Specific constraints such as the heavy neck, low and anterior hyoid position, lack of chin projection, and deficient platysma tone are addressed here in the scope of cervical rejuvenation.

Lastly, the skin represents the most conspicuous marker of the aging neck. With aging, the collagen and elastin fibers degenerate and the skin is no longer able to adhere to soft tissue contours of the neck. Redundancy and sagging of the neck skin leads to effacement of the cervicomental angle and cervical rhytids, which can contribute to the appearance of the heavy neck. Loss of organization of collagen fibers also reduces the skins ability to conform, tighten, and contract in response to surgical maneuvers.

\section{The Difficult Neck}

Between the extremes of the "perfect" result in rhytidectomy, seen in the patient with ideal characteristics, and the unacceptable result in the patient who was a poor candidate and addressed with poor technique, lies a group of patients who present considerable challenges but can be considered candidates for an acceptable result. The difficult neck is one that the surgeon strives to get a good result in spite of having less than ideal anatomic characteristics. These patients will achieve these "acceptable" results through the best analysis of their deficiencies and the application of the best methods to correct these deficiencies. "Acceptable" results also require good preoperative communication with the patient about the limitations inherent in their anatomy and technique.

Common issues that present challenges include the following: large amounts of inelastic skin, the heavy neck, platysma bands, microgenia, hyoid malposition, ptotic submandibular glands, and digastric hypertrophy. Rhytidectomy in the male patient also presents challenges due to the characteristic heavier skin of the bearded. ${ }^{48,49}$ The focus in this paper will be mainly on patients with a heavy neck secondary to an excess of adipose tissue. Other issues to be discussed briefly will be management of inadequate chin projection, platysma bands, as well as an excess of skin. In general, these patients can still be considered reasonable candidates for rhytidectomy. It must be noted that certain modifications to the technique need to be performed to achieve acceptable results, and again, these patients have to be counseled preoperatively as to expectations. These modifications in technique include the correction of underlying skeletal features, lipectomy, the creation of substantial SMAS flaps, and wide skin undermining to reposition and remove abundant skin. None of the techniques is novel but instead require an individualization of technique to each patient.

\section{Challenges Encountered and Their Solutions}

\section{Skin}

The amount of excess and elasticity of the skin should be ascertained as this determination will have a direct influence on incision design, particularly in the postauricular area. Laxity of skin, especially of inelastic skin, will have to be managed similarly to that of patients with thinner necks. In general, patients with a large excess of skin that is inelastic will require a longer skin incision to achieve the appropriate vector of movement and removal of the skin. The age of the patient will also have an impact here; in general, the older patient will have less elastic skin, and again, will require a longer incision to manage skin excess and removal. As a general observation, the heavier the neck in the older patient with inelastic skin, the longer the incision will have to be. In the younger patient with elastic skin, a short-scar approach can be considered. To allow adequate skin redraping, wider undermining may also be necessary in the patient with a heavy neck (-Fig. 4).

\section{Adipose}

The heavy neck is becoming an increasingly common challenge in rhytidectomy with increasing population weight norms, as well as with larger numbers of ethnic patients that impart different body shapes. Given the heavy neck secondary to an excess of subcutaneous adipose and subplatysmal adipose, patient weight loss does little to impact the shape of the neck without significant surgical intervention. The patient presents with an anatomic situation in which some of the more desirable features of the aesthetic neck will be less likely to be achieved. The treatment strategy should delineate an approach in setting expectations, as well as the surgical maneuvers to be performed.

The amount and distribution of adipose should be ascertained and whether or not the fat is subcutaneous and/or subplatysmal. Depending on the location, a decision can be made about reduction via direct lipectomy, as is performed in the submental area, or with liposuction, laser-assisted lipolysis, or other adjunct technique. Direct submental lipectomy may have to be performed in the patient with a severe obtuse cervicomental angle. The lipectomy should be performed cautiously to avoid skeletonizing the submental area and creating a "cobra" deformity. Ultrasonic- and laser-assisted lipolysis are advocated by some authors but are not universally accepted to be superior to conventional suction-assisted lipectomy in this anatomic area. ${ }^{11,50}$ The senior author (FGF), to date, has acceptable results from conventional liposuction and direct lipectomy in the setting of rhytidectomy. Some of the undermining of the flaps may also be accomplished with the liposuction cannula, thus allowing greater mobility of the skin flaps while maintaining some of the vascular connections with underlying structures (-Fig. 5).

\section{Skeletal Deficiencies}

The skeletal features are examined. This determination is among the most important variables to be considered in the 
A
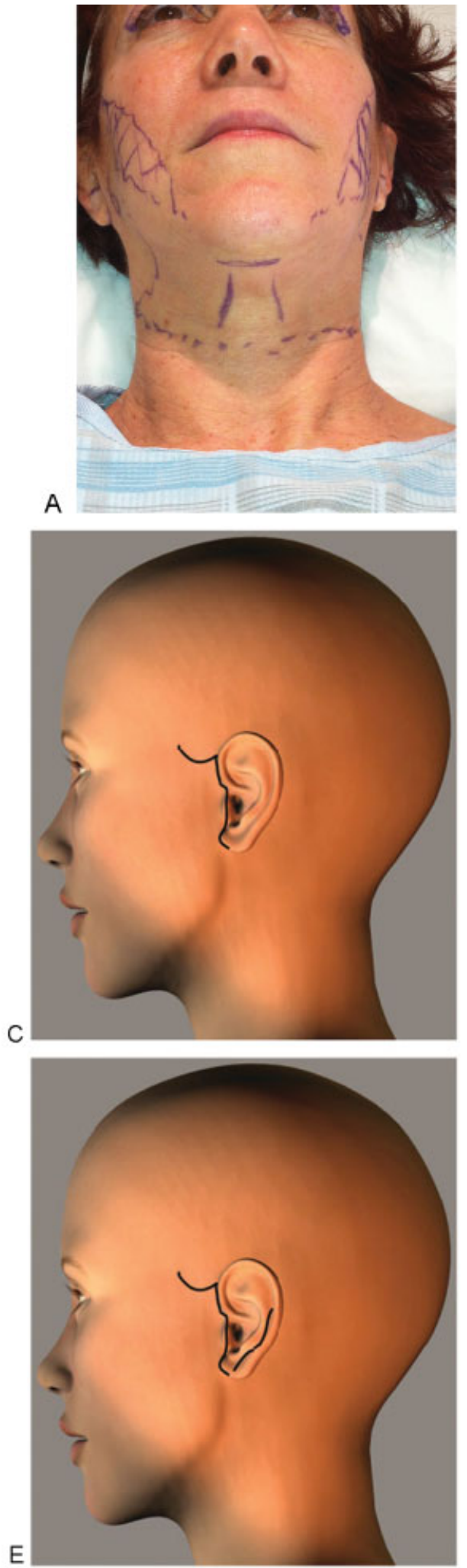
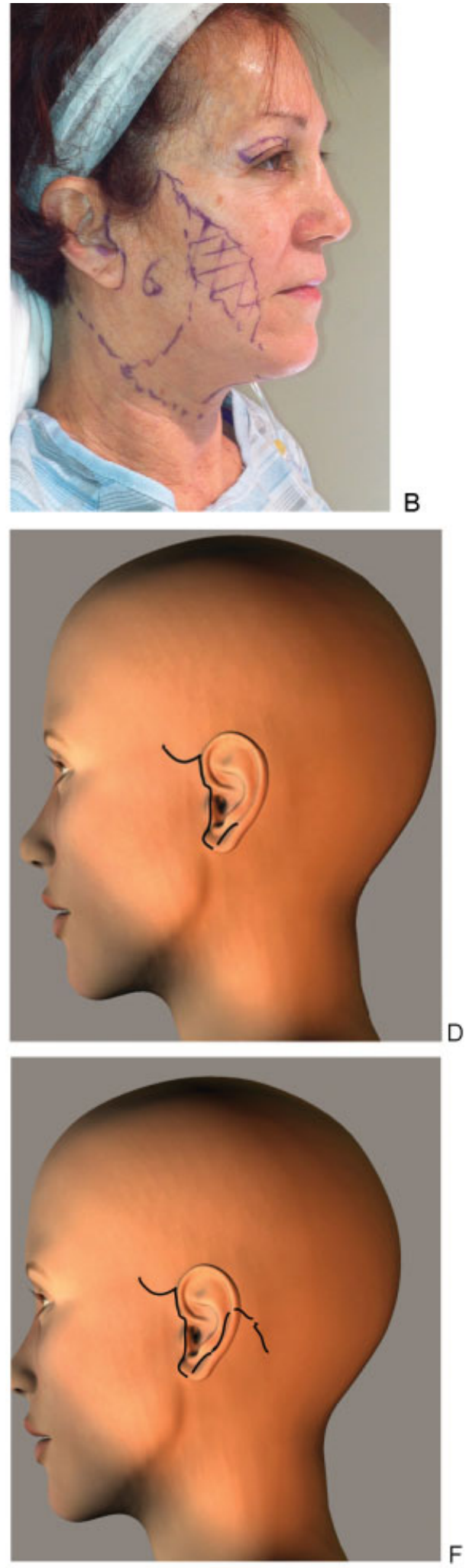

Fig. 4 Clinical photographs and drawings depicting skin incisions and areas of undermining for lower rhytidectomy. (A, B) Clinical photograph showing periauricular-posttragal incision plan. On the lateral, projection is depicted the extent of a $6 \mathrm{~cm}$ flap as might be performed anteriorly during a short scar rhytidectomy. Also is depicted the more extensive undermining and skin elevation anteriorly and into the neck necessary in patients with a more challenging neck. The position of her platysma bands is marked. (C-F) Drawing depicting the individualization of incisions design possible to allow adaptation to a patients anatomy. (G) Drawing depicting the more extensive soft tissue mobilization possible with traditional long incision techniques compared with short incision techniques. 

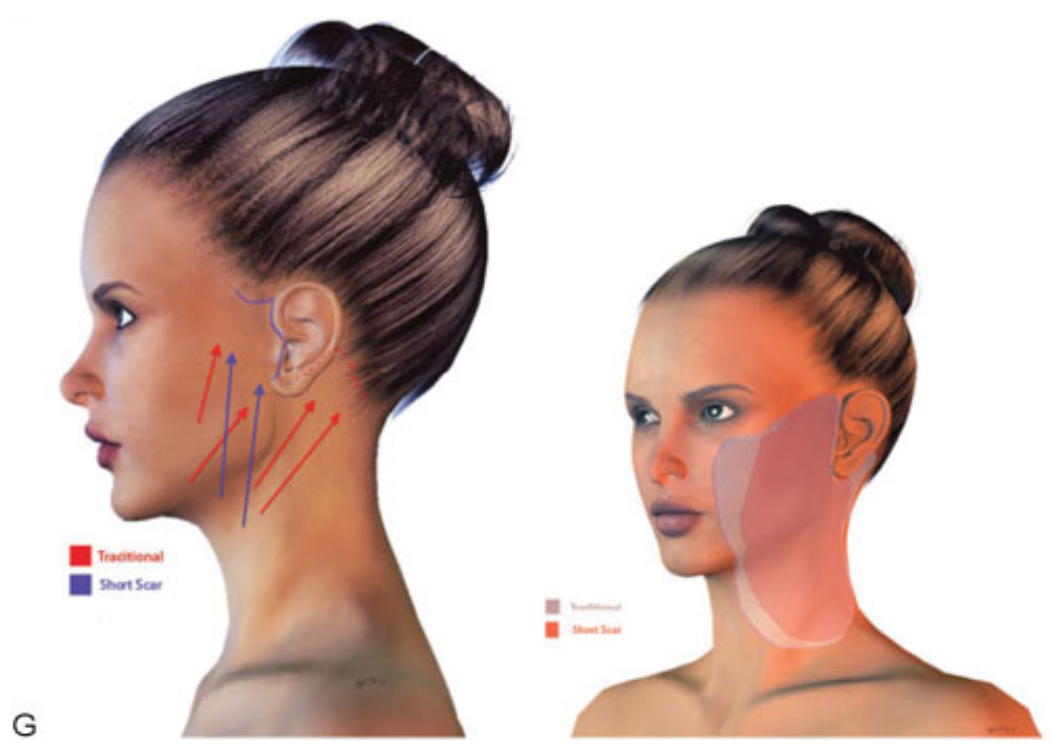

Fig. 4 (Continued)

establishment of an acceptable jaw line and cervicomental angle. The underlying skeletal features are the foundation upon which the overlying soft tissues can be redraped and repositioned. The underlying anatomy creates the form; the skin serves as the cover. Through relatively simple maneuvers (e.g., genioplasty and the use of other implants), the skeletal features can be significantly improved. In the situation of the heavy neck, this improvement of the skeletal features may be even more important to impart definition along the jaw line. Where possible, microgenia should be corrected. In addition, the contour of the jawline can be augmented with injectable fillers and implants (-Fig. 6).

\section{Superficial Musculoaponeurotic System}

The consideration and management of the SMAS or layer is among the most important aspects of rhytidectomy. For an in-depth review of this topic, the reader is referred to other references. $^{20,24,31,32,34,36,42,43,51-59}$ In examining the topic, what becomes very clear is that there is a variety of methods advocated to "tighten," "lift," "advance," and "reposition" this very important layer. The methods advocated range from those that superficially and in a limited fashion plicate and imbricate the SMAS, to those that involve a more extensive flap development with a multivector fixation, to those that advocate a deep plane dissection. While there is limited consensus about which techniques have a greater long-term advantage, there appears to be a general consensus that the vector of advancement should be posterior and superior to favorably affect the midface, the jowls, and the neck. There is also broad agreement that some form of SMAS management should be considered in most rhytidectomy cases. The senior author has utilized several of these methods across patients with varying characteristics. In general, for the more challenging neck, and particularly the heavier neck, a more extensive SMAS flap is developed to mobilize, advance, and support the heavier tissues. The exact technique used is individualized to the particular patient (-Fig. 7).

\section{Platysma Bands}

The presence of platysma bands, while less visible in these patients with heavier necks, will still have an important impact on the final result. In addition, and possibly most importantly, the cervicomental angle must be optimized if patients with heavy necks are to be offered the best possible results with rhytidectomy.

Platysmaplasty should be done when there is evidence of platysma bands, as this will be necessary to improve the cervicomental angle. Similar to other situations when the midline platysma are sutured together, even with the heavy neck, optimal skin redraping may require the development of a long flap for undermining from ear to ear. In some patients with particularly heavy tissues, a form of a sling or a suspension is advocated to maximize the cervicomental angle., 94,60

\section{Secondary Procedure}

Adequate patient counseling requires informing the patient that they may require a secondary procedure or tuck-up approximately 1 year after their primary procedure to optimize their result (-Fig. 8).

\section{Further Adjuncts and Techniques}

\section{Use of Fat and Fillers}

As the focus of this paper is largely on the patient with a heavier neck, the use of fillers is limited if the consideration is the soft tissues of the neck. In contrast, fillers can be used to fill in deficiencies of the mandible and create improvement in the geniomandibular groove and angle.

The use of injectable fillers or structural fat grafting to improve the appearance of volume loss in the aging face and 
A
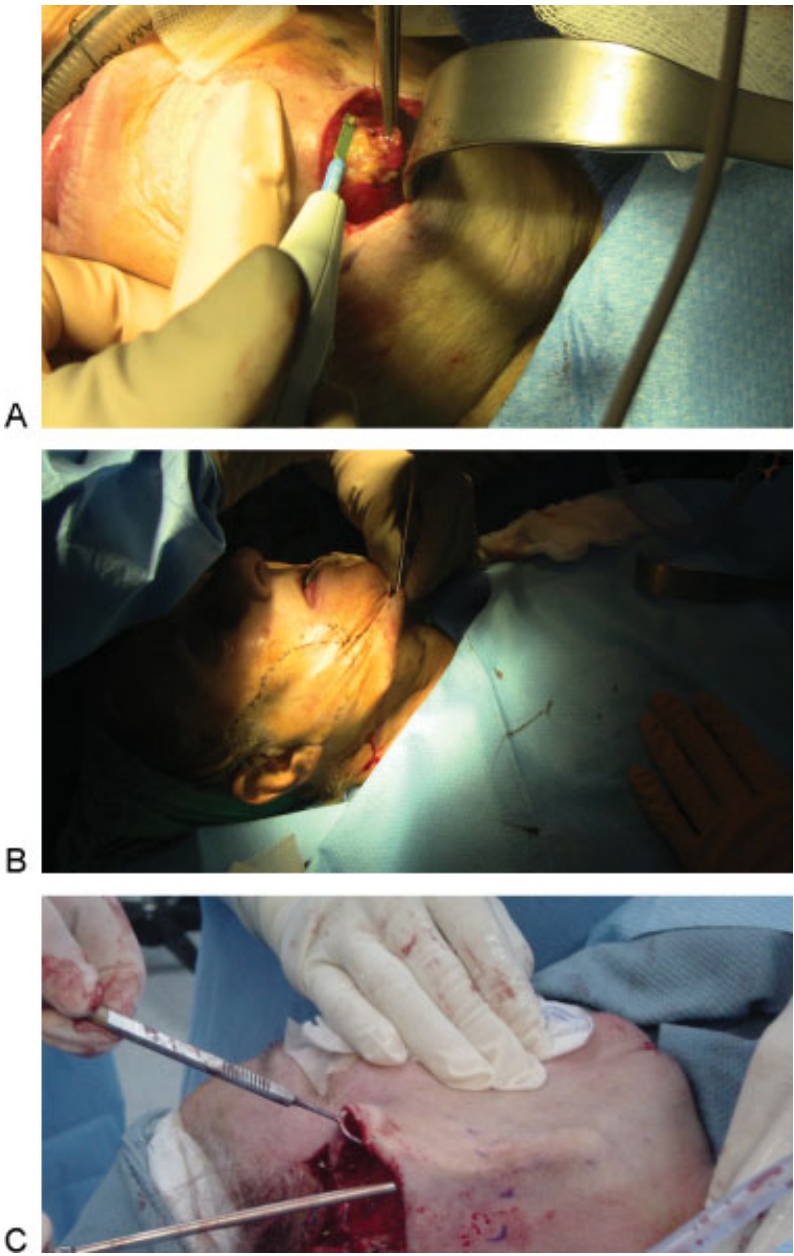

Fig. 5 Clinical photographs of fat removal. (A) Direct submental lipectomy. (B) Suction-assisted lipectomy carried through submental incision to debulk the submental area and undermine the skin flap more laterally. (C) Open suction lipectomy to facilitate and extend flap undermining.

neck has been described and has demonstrated some success. ${ }^{61}$ The use of injectable filler materials, such as hyaluronic acid, human-derived collagen, calcium hydroxyapatite, polymethyl methacrylate, and poly-L-lactic acid have long been regarded for their use in the correction of tissue atrophy related to facial aging. They have rapid, predictable results, demonstrate a relative ease of delivery, and have a favorable safety profile. One of the most common applications of facial fillers in the treatment of the aging neck is to improve the appearance of the prejowl sulcus and the jawline. ${ }^{62}$ There have been reports of successful results in the literature with both the use of hyaluronic acid fillers and poly-L-lactic acid. ${ }^{63-65}$ While these fillers are generally quite safe, it is important to be familiar with their potential complications and their management. Although rare, complications such as cellulitis, granuloma formation, or skin necrosis have the potential to cause severe and possibly permanent scarring.

Autologous fat transfer has similarly been described. Contouring the prejowl sulcus is considered one of the most important applications of autologous fat transfer. ${ }^{63,66}$ One technique described in the literature, which has had
A

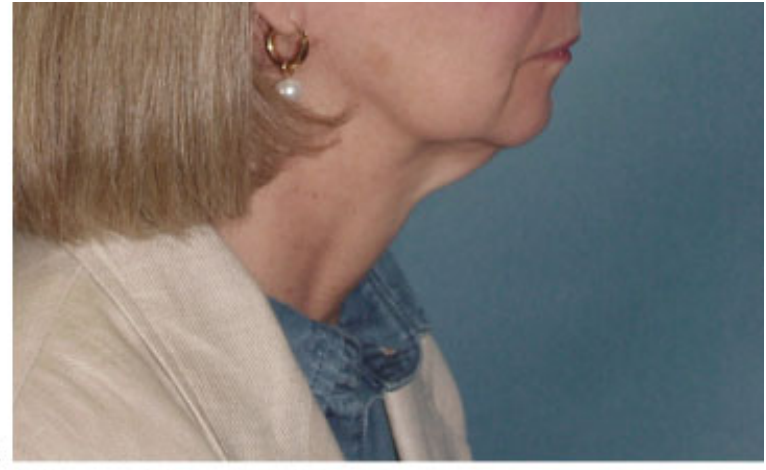

B

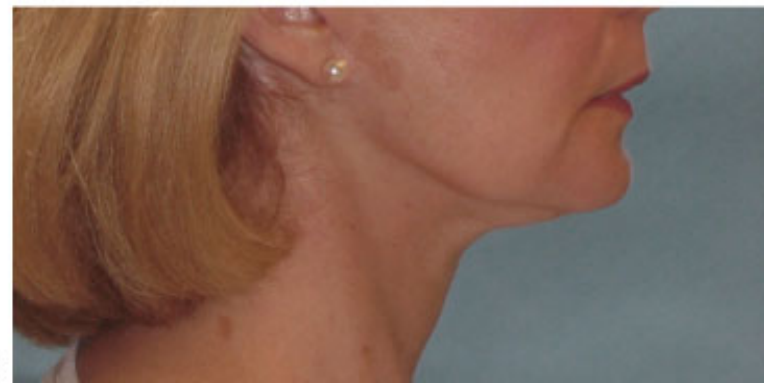

Fig. 6 Clinical photograph demonstrating the improvement of this patient's skeletal features with chin augmentation thus facilitating the creation of a desirable mentocervical angle with rhytidectomy (A) Prior to chin augmentation and facelift. (B) Postoperative photograph after chin augmentation and facelift.

success, makes use of three sites for injection of the autologous fat: along the anterior surface of the mandible along the periosteum, along the inferior surface of the mandible and toward the digastric muscle, and lastly, obliquely between those sites in the superficial tissues. ${ }^{66}$ The possibility of fat resorption remains a major concern with the use of autologous fat transfer, and changes in the appearance of the fat are also possible if patients undergo any significant weight loss or gain after their procedure. A recent study, for example, found that only $31.8 \%$ of the original volume replaced was retained at 16 months posttreatment with $24 \%$ of patients electing to undergo additional fat transfer within the first year. ${ }^{63}$

\section{Resurfacing, Lasers, and Chemical Peels}

The quality and texture of the neck skin is an important component in the appearance of a youthful neck. Changes in skin texture, skin laxity, and the presence of rhytids all contribute to the appearance of the aging neck. Patients often present with dermal changes, such as irregular pigmentation, lentigines, keratosis, wrinkling, and striae distensae, which are amenable to treatment with resurfacing. There are descriptions of various resurfacing modalities in the literature that have been used successfully to improve these signs of aging in the neck, including dermabrasion, chemical peels, and laser resurfacing.

The use of lasers with different wavelengths allows one to target different chromophores, such as water, melanin, or hemoglobin to achieve the desired clinical result. The lasers most commonly used for resurfacing of the neck include the 


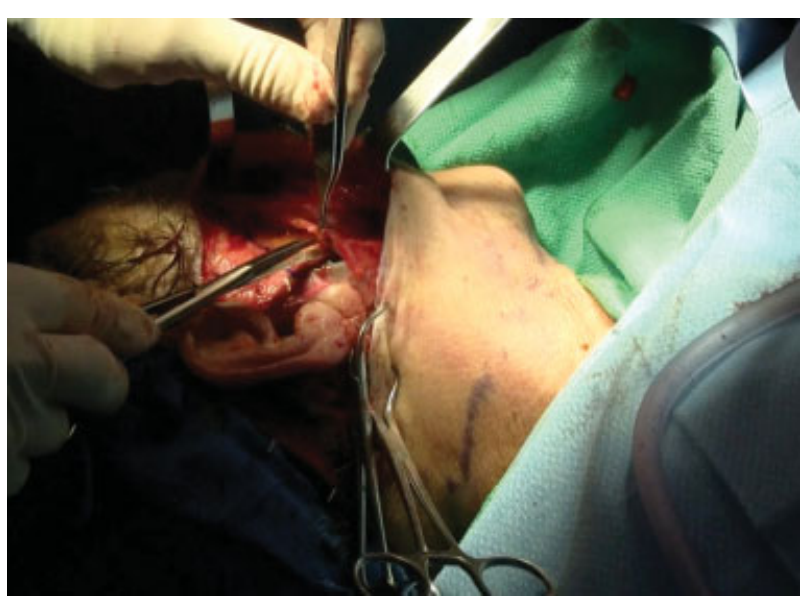

Fig. 7 Clinical photograph depicting elevation of superficial musculoaponeurotic system flap.

$\mathrm{CO}_{2}$ laser and the erbium: yttrium-aluminum-garnet laser. The targeted chromophore for these lasers is water located in the dermis. A study of 10 patients who underwent between one and three sessions of non ablative $\mathrm{CO}_{2}$ laser resurfacing of the neck found significant improvements in the appearance of skin texture, skin laxity, and rhytids, with an average of 1.4 sessions required with no reports of adverse reaction or complication. ${ }^{67}$ Laser resurfacing of the neck, however, should be performed with caution as complications may occur. ${ }^{68}$

Another method for resurfacing the neck to improve the signs of aging is the use of chemical peels. Although not as well described in the literature as the use of chemical peels for the facial skin, some authors have reported success addressing skin texture, irregular pigmentation, rhytids, lentigines, and actinic keratosis with chemical peeling of the neck. ${ }^{69}$ Again, caution is advocated so as to avoid complications.

The following patients presented with anatomic challenges managed with the techniques described.

\section{Patient 1}

This patient presented with the following characteristics: excessive thick inelastic skin, moderate adipose, significant jowling, and platysma bands (- Fig. 9).

\section{Patient 2}

This patient presented with the following characteristics: excessive thick inelastic skin, moderate adipose, significant jowling, and minor wide platysma bands (-Fig. 10).

\section{Patient 3}

This patient presented with the following characteristics: excessive thick inelastic skin, moderate adipose, significant jowling, minor wide platysma bands, skeletal deficiency with marked microgenia. This patient's history was complicated by a past left neck dissection (-Fig. 11).
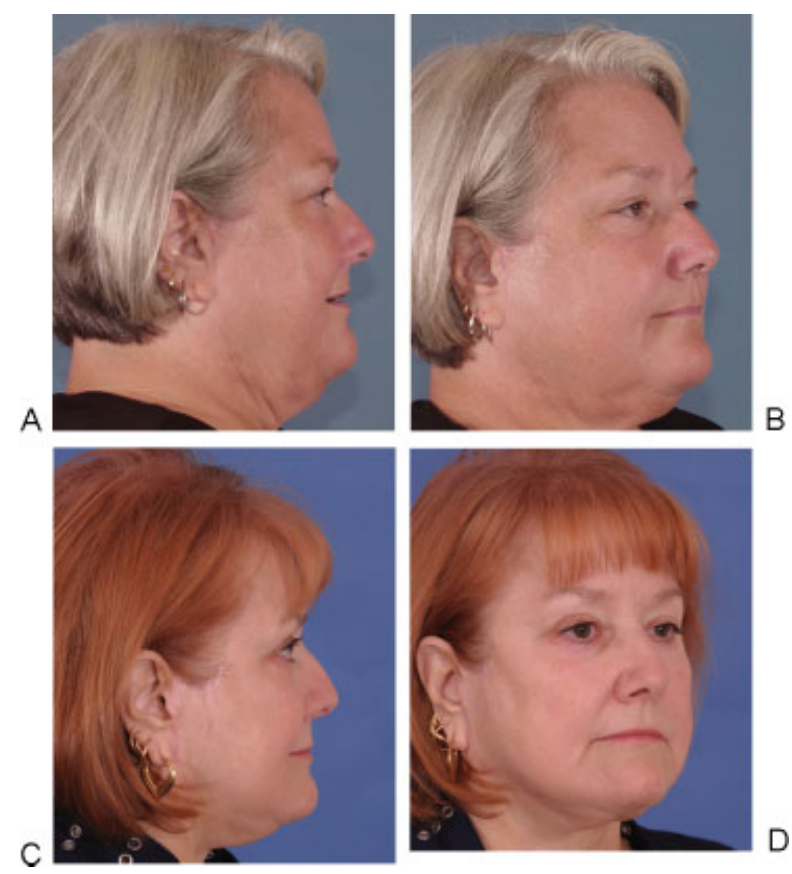

Fig. 8 Clinical photographs of patient with difficult neck anatomy who had undergone previous short scar lower facelift at another office (within 24 months) and improvement after in-office tuck-up procedure involving minimal skin excision and purse-string tightening of superficial musculoaponeurotic system. (A, B) Before tuck-up. (C, D) After tuck-up. 


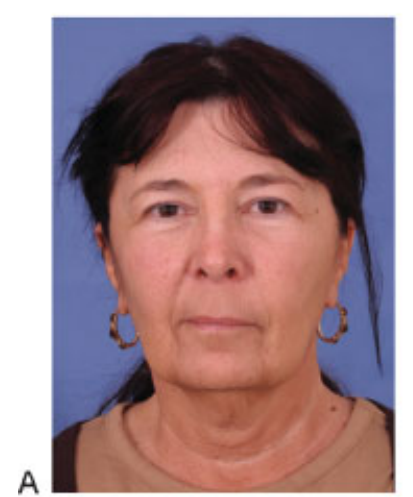

A
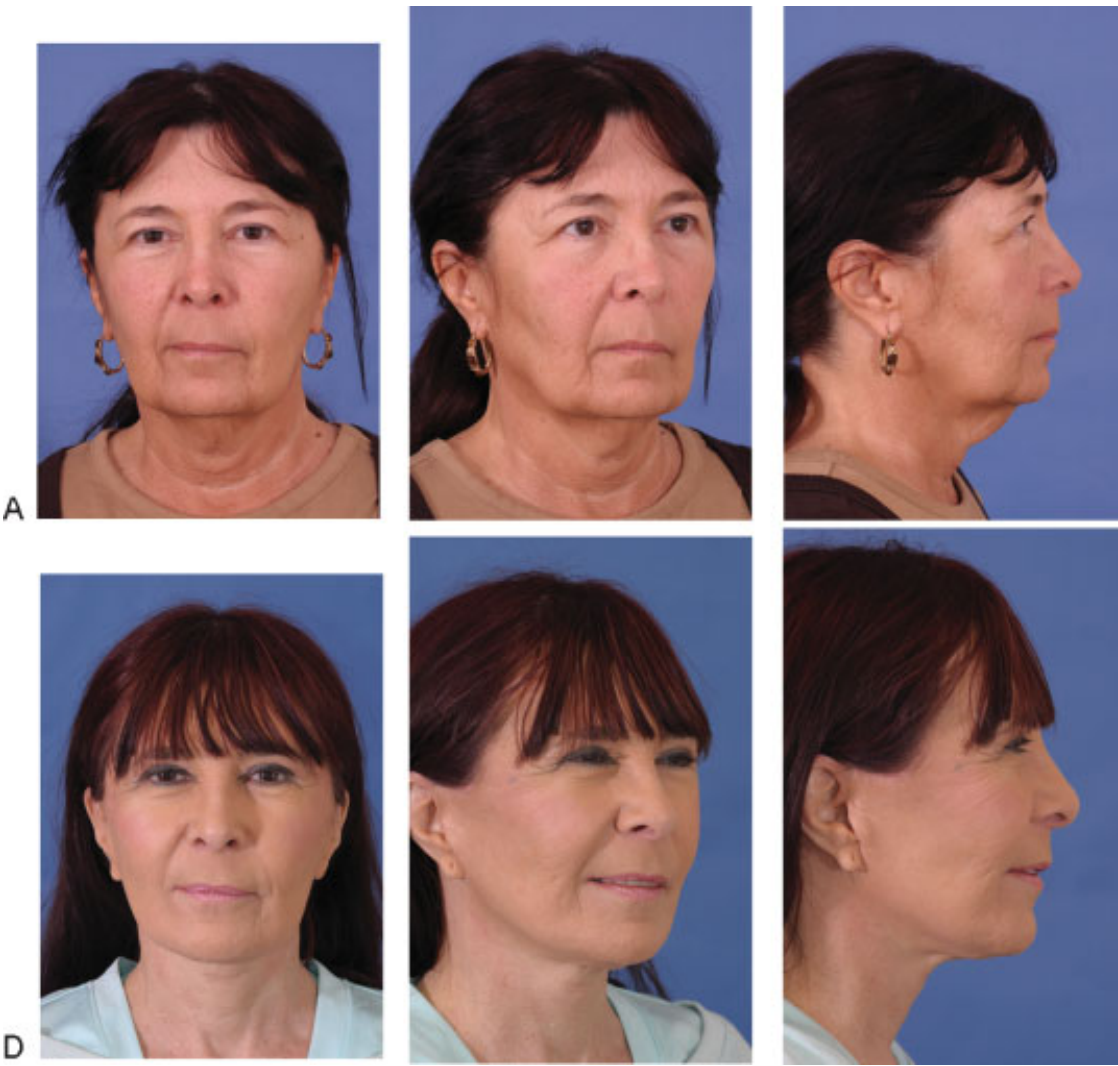

$B, C$

$E, F$

Fig. 9 Clinical photographs with the following anatomic challenging characteristic who presented for facelift. Significant excessive thick inelastic skin, moderate adipose, significant jowling, and platysma bands. Operative approach included the following: traditional long incisions with postauricular extension, wide undermining across neck with release of mandibular ligament, liposuction, management of platysma with anterior suturing and lateral suspension, superficial musculoaponeurotic system was managed by creation of limited flap and imbrication. (A-C) Preoperative. (D-F) Postoperative.
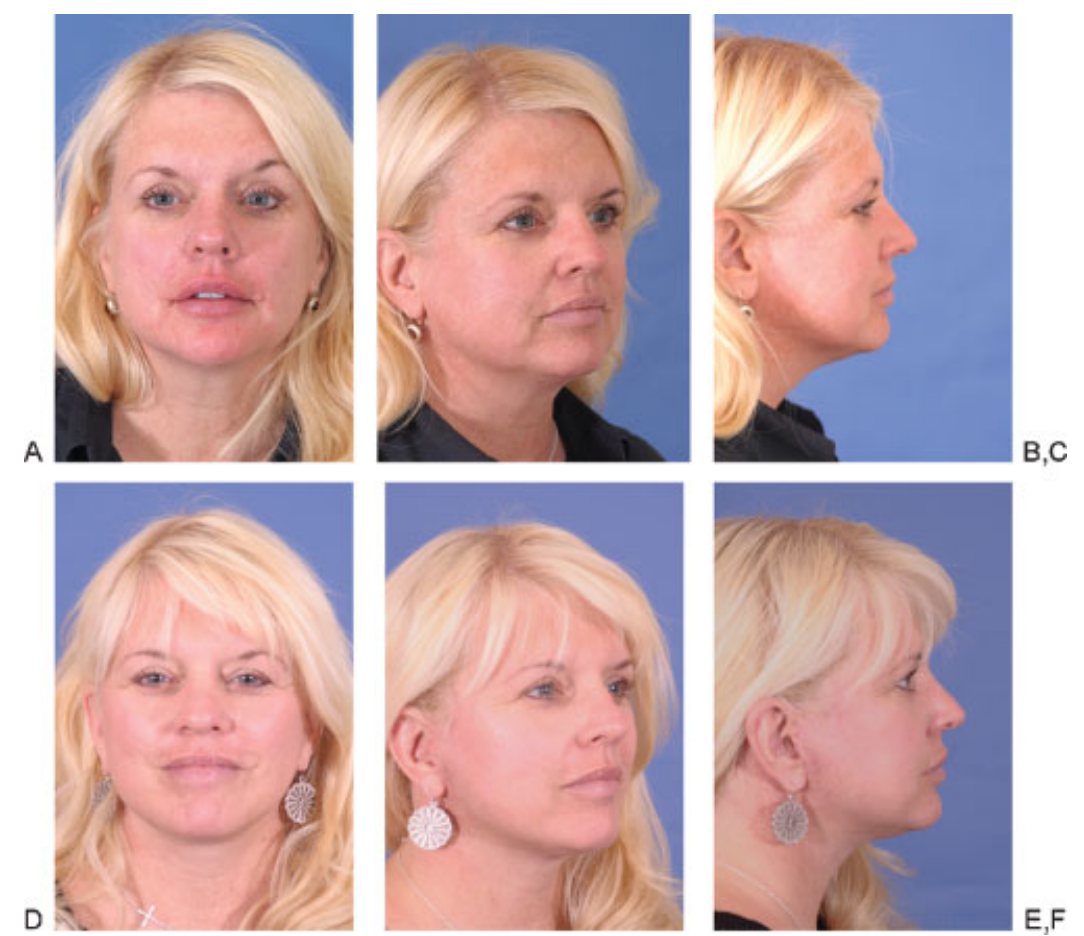

Fig. 10 Clinical photographs with the following anatomic challenging characteristic who presented for facelift. Significant excessive thick inelastic skin, moderate adipose, significant jowling, and minor wide platysma bands. Operative approach included the following: traditional long incisions with postauricular extension, wide undermining across neck with release of mandibular ligament, liposuction, management of platysma with lateral suspension, superficial musculoaponeurotic system was managed by creation of extended flap and imbrication. (A-C) Preoperative. (D-F) Postoperative. 

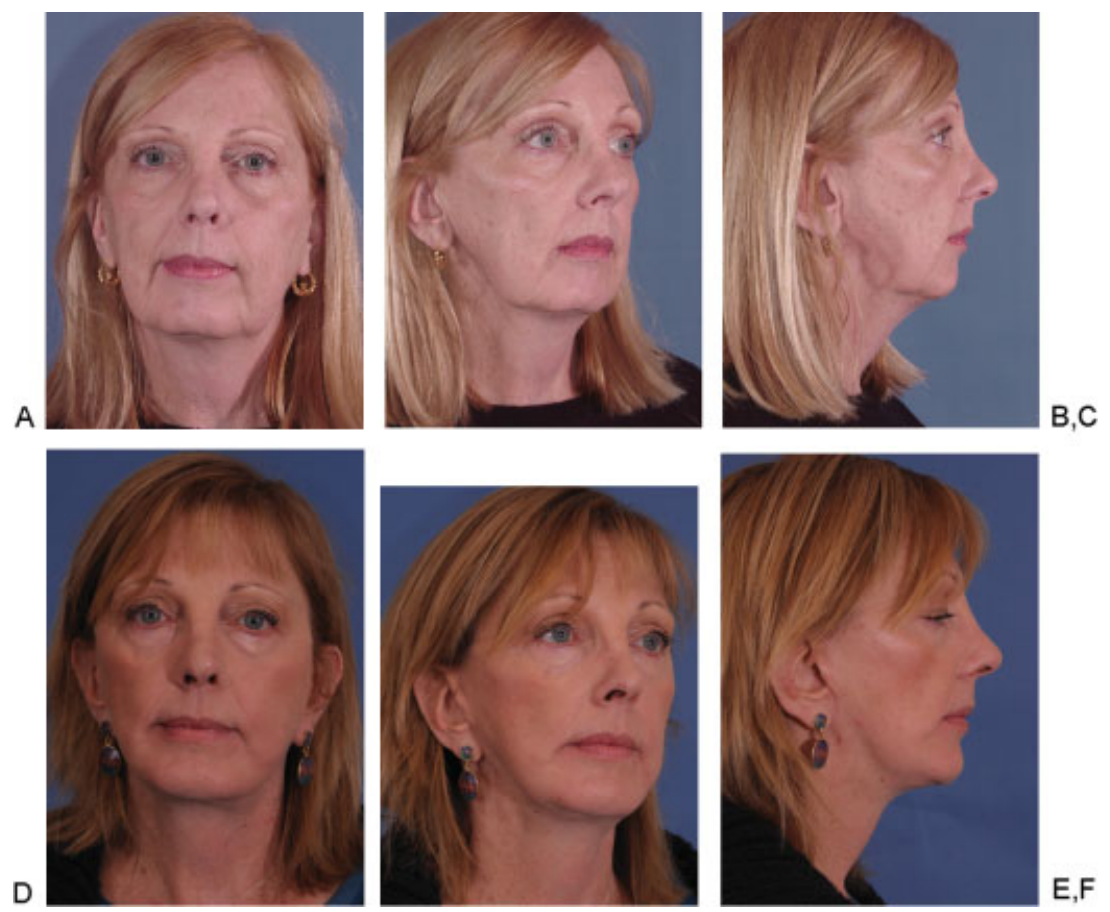

Fig. 11 Clinical photographs with the following anatomic challenging characteristic who presented for facelift. Significant excessive thick inelastic skin, moderate adipose, significant jowling and minor wide platysma bands, skeletal deficiency with marked microgenia. This patient's history complicated by past left neck dissection. Operative approach included the following: traditional long incisions with postauricular extension, cautious wide undermining with release of mandibular ligament, liposuction, management of platysma with anterior suturing and lateral suspension, superficial musculoaponeurotic system was managed by creation of limited flap (with respect to left side) and imbrication. This patient required tuck-up of left-side skin 1 year after primary surgery. (A-C) Preoperative. (D-F) Postoperative, 1 year after tuck-up.

\section{Summary}

The difficult neck is one that the surgeon strives to get a good result in spite of the patient having less than ideal anatomic characteristics. Acceptable results can be realized through the best analysis of their deficiencies and the application of the best methods to correct these deficiencies. The methods employed are those that are based on established principles and application. In the case of the difficult neck, the application is individualized and appropriately modified to the patient's anatomy.

\section{Acknowledgment \\ The authors wholeheartedly thank Kim Gordon for edito- rial assistance.}

\section{References}

1 AAFPRS. 2012 AAFPRS Membership Study. International Communications Research. Media, PA 2013

2 McCollough EG, Ha CD. The McCollough Facial Rejuvenation System: expanding the scope of a condition-specific algorithm. Facial Plast Surg 2012;28(1):102-115

3 Langsdon P, Shires C, Gerth D. Lower face-lift with extensive neck recontouring. Facial Plast Surg 2012;28(1):89-101

4 Marchac D. Julien Bourguet. The pioneer in aesthetic surgery of the neck. Clin Plast Surg 1983;10(3):363-365
5 Skoog T. Plastic surgery: the aging face. In: Skoog TG, ed. Plastic Surgery: New Methods and Refinements. Philadelphia, PA: WB Saunders; 1974:300-330

6 Mitz V. The superficial musculoaponeurotic system: a clinical evaluation after 15 years of experience. Facial Plast Surg 1992; $8(1): 11-17$

7 Mitz V, Peyronie M. The superficial musculo-aponeurotic system (SMAS) in the parotid and cheek area. Plast Reconstr Surg 1976; 58(1):80-88

8 Millard DR Jr, Garst WP, Beck RL, Thompson ID. Submental and submandibular lipectomy in conjunction with a face lift, in the male or female. Plast Reconstr Surg 1972;49(4):385-391

9 Connell BF. Contouring the neck in rhytidectomy by lipectomy and a muscle sling. Plast Reconstr Surg 1978;61(3):376-383

10 Courtiss EH. Suction lipectomy of the neck. Plast Reconstr Surg 1985;76(6):882-889

11 Grotting JC, Beckenstein MS. Cervicofacial rejuvenation using ultrasound-assisted lipectomy. Plast Reconstr Surg 2001;107(3): 847-855

12 Gryskiewicz JM. Submental suction-assisted lipectomy without platysmaplasty: pushing the (skin) envelope to avoid a face lift for unsuitable candidates. Plast Reconstr Surg 2003;112(5): 1393-1405, discussion 1406-1407

13 McMenamin P. Laser face-lifts: a new paradigm in face-lift surgery. Facial Plast Surg 2011;27(4):299-307

14 Morrison W, Salisbury M, Beckham P, Schaeferle M III, Mladick R, Ersek RA. The minimal facelift: liposuction of the neck and jowls. Aesthetic Plast Surg 2001;25(2):94-99

15 Teimourian B. Face and neck suction-assisted lipectomy associated with rhytidectomy. Plast Reconstr Surg 1983;72(5):627-633

16 Feldman JJ. Corset platysmaplasty. Plast Reconstr Surg 1990;85(3): 333-343 
17 Conley J. Face-lift operation. N Y State J Med 1971;71(15): 1819-1826

18 Zide BM, Laub DR. Variations of technique in the face-lift operation. Ann Plast Surg 1979;2(2):114-120

19 Deutsch HL. Resection of platysma bands in face-lift surgery. J Dermatol Surg Oncol 1980;6(12):1003-1006

20 Owsley JQ Jr. SMAS-platysma facelift. A bidirectional cervicofacial rhytidectomy. Clin Plast Surg 1983;10(3):429-440

21 Webster RC, Smith RC, Smith KF. Face lift, part 2: Etiology of platysma cording and its relationship to treatment. Head Neck Surg 1983;6(1):590-595

22 Connell BF, Shamoun JM. The significance of digastric muscle contouring for rejuvenation of the submental area of the face. Plast Reconstr Surg 1997;99(6):1586-1590

23 Sullivan PK, Freeman MB, Schmidt S. Contouring the aging neck with submandibular gland suspension. Aesthet Surg J 2006;26(4): 465-471

24 Adamson PA, Dahiya R, Litner J. Midface effects of the deep-plane vs the superficial musculoaponeurotic system plication face-lift. Arch Facial Plast Surg 2007;9(1):9-11

25 Gladstone GJ, Myint S, Black EH, Brazzo BG, Nesi FA. Fundamentals of facelift surgery. Ophthalmol Clin North Am 2005;18(2): 311-317, vii

26 Hamra ST. The tri-plane face lift dissection. Ann Plast Surg 1984; 12(3):268-274

27 Marten TJ. High SMAS facelift: combined single flap lifting of the jawline, cheek, and midface. Clin Plast Surg 2008;35(4):569-603, vi-vii

28 Massiha H. Short-scar face lift with extended SMAS platysma dissection and lifting and limited skin undermining. Plast Reconstr Surg 2003;112(2):663-669

29 Miller TR, Eisbach KJ. SMAS facelift techniques to minimize stigmata of surgery. Facial Plast Surg Clin North Am 2005;13(3): 421-431

30 Mottura AA. SPA face lift: SMAS plication-anchoring. Aesthetic Plast Surg 2011;35(4):511-515

31 Owsley JQ Jr. SMAS-platysma face lift. Plast Reconstr Surg 1983; 71(4):573-576

32 Kamer FM, Frankel AS. SMAS rhytidectomy versus deep plane rhytidectomy: an objective comparison. Plast Reconstr Surg 1998; 102(3):878-881

33 Kamer FM, Parkes ML. The two-stage concept of rhytidectomy. Trans Sect Otolaryngol Am Acad Ophthalmol Otolaryngol 1975; 80(6):546-550

34 McCollough EG, Perkins SW, Langsdon PR. SASMAS suspension rhytidectomy. Rationale and long-term experience. Arch Otolaryngol Head Neck Surg 1989;115(2):228-234

35 Perkins SW, Patel AB. Extended superficial muscular aponeurotic system rhytidectomy: a graded approach. Facial Plast Surg Clin North Am 2009;17(4):575-587, vi

36 Hamra ST. The deep-plane rhytidectomy. Plast Reconstr Surg 1990;86(1):53-61, discussion 62-63

37 Dedo DD. "How I do it"-plastic surgery. Practical suggestions on facial plastic surgery. A preoperative classification of the neck for cervicofacial rhytidectomy. Laryngoscope 1980;90(11 Pt 1):1894-1896

38 Ellenbogen R, Karlin JV. Visual criteria for success in restoring the youthful neck. Plast Reconstr Surg 1980;66(6):826-837

39 Baker DC, Nahai F, Massiha H, Tonnard P. Short scar face lift. Aesthet Surg J 2005;25(6):607-617

40 Jacono AA, Rousso JJ. The modern minimally invasive face lift: has it replaced the traditional access approach? Facial Plast Surg Clin North Am 2013;21(2):171-189

41 Fedok FG, Sedgh J. Managing the neck in the era of the short scar face-lift. Facial Plast Surg 2012;28(1):60-75

42 Baker DC. Minimal incision rhytidectomy (short scar face lift) with lateral SMASectomy: evolution and application. Aesthet Surg J 2001;21(1):14-26
43 Jacono AA, Parikh SS. The minimal access deep plane extended vertical facelift. Aesthet Surg J 2011;31(8):874-890

44 Giampapa VC, Di Bernardo BE. Neck recontouring with suture suspension and liposuction: an alternative for the early rhytidectomy candidate. Aesthetic Plast Surg 1995;19(3): 217-223

45 Liu TS, Owsley JQ. Long-term results of face lift surgery: patient photographs compared with patient satisfaction ratings. Plast Reconstr Surg 2012;129(1):253-262

46 de Castro CC. The anatomy of the platysma muscle. Plast Reconstr Surg 1980;66(5):680-683

47 Gonzalez-Ulloa M. Quantitative principles in cosmetic surgery of the face (profileplasty). Plast Reconstr Surg Transplant Bull 1962; 29:186-198

48 Lawson W, Naidu RK. The male facelift. An analysis of 115 cases. Arch Otolaryngol Head Neck Surg 1993;119(5):535-539, discussion 540-541

49 Papel ID, Lee E. The male facelift: considerations and techniques. Facial Plast Surg 1996;12(3):257-263

50 Stebbins WG, Hanke CW. Rejuvenation of the neck with liposuction and ancillary techniques. Dermatol Ther 2011;24(1):28-40

51 Webster RC, Smith RC, Smith KF. Face lift, Part 4: Use of superficial musculoaponeurotic system suspending sutures. Head Neck Surg 1984;6(3):780-791

52 Webster RC, Smith RC, Smith KF. Face lift, Part 3: Plication of the superficial musculoaponeurotic system. Head Neck Surg 1983; 6(2):696-701

53 Waterhouse N, Vesely M, Bulstrode NW. Modified lateral SMASectomy. Plast Reconstr Surg 2007;119(3):1021-1026, discussion 1027-1028

54 Teimourian B, Delia S, Wahrman A. The multiplane face lift. Plast Reconstr Surg 1994;93(1):78-85

55 Stocchero IN. Shortscar face-lift with the RoundBlock SMAS treatment: a younger face for all. Aesthetic Plast Surg 2007; 31(3):275-278

56 Randall P, Skiles MS. The "SMAS sling": an additional fixation in face lift surgery. Ann Plast Surg 1984;12(1):5-9

57 Panfilov DE. MIDI face-lift and tricuspidal SMAS-flap. Aesthetic Plast Surg 2003;27(1):27-37

58 Berry MG, Davies D. Platysma-SMAS plication facelift. J Plast Reconstr Aesthet Surg 2010;63(5):793-800

59 Becker FF, Bassichis BA. Deep-plane face-lift vs superficial musculoaponeurotic system plication face-lift: a comparative study. Arch Facial Plast Surg 2004;6(1):8-13

60 Prabhat A, Dyer WK II. Improving surgery on the aging neck with an adjustable expanded polytetrafluoroethylene cervical sling. Arch Facial Plast Surg 2003;5(6):491-501

61 Ransom ER, Antunes MB, Bloom JD, Greco T. Concurrent structural fat grafting and carbon dioxide laser resurfacing for perioral and lower face rejuvenation. J Cosmet Laser Ther 2011;13(1):6-12

62 Winters R, Moulthrop T. Is autologous fat grafting superior to other fillers for facial rejuvenation? Laryngoscope 2013;123(5): 1068-1069

63 Meier JD, Glasgold RA, Glasgold MJ. Autologous fat grafting: longterm evidence of its efficacy in midfacial rejuvenation. Arch Facial Plast Surg 2009;11(1):24-28

64 Carruthers JD, Carruthers A. Facial sculpting and tissue augmentation. Dermatol Surg 2005;31(11 Pt 2):1604-1612

65 Moy RL, Fincher EF. Poly-L-lactic acid for the aesthetic correction of facial volume loss. Aesthet Surg J 2005;25(6):646-648

66 Glasgold M, Lam SM, Glasgold R. Autologous fat grafting for cosmetic enhancement of the perioral region. Facial Plast Surg Clin North Am 2007;15(4):461-470, vi

67 Hollmig ST, Struck SK, Hantash BM. Establishing the safety and efficacy of simultaneous face lift and intraoperative full face and neck fractional carbon dioxide resurfacing. Plast Reconstr Surg 2012;129(4):737e-739e 
450 The Difficult Neck in Facelifting Fedok et al.

68 Avram MM, Tope WD, Yu T, Szachowicz E, Nelson JS. Hypertrophic scarring of the neck following ablative fractional carbon dioxide laser resurfacing. Lasers Surg Med 2009;41(3): $185-188$
69 Cook KK, Cook WR Jr. Chemical peel of nonfacial skin using glycolic acid gel augmented with TCA and neutralized based on visual staging. Dermatol Surg 2000;26(11):994-999 